\title{
PROPIEDADES FISICOQUÍMICAS, FUNCIONALES Y ESTRUCTURALES DE ALMIDONES NATIVOS Y ACETILADOS OBTENIDOS A PARTIR DE LA PAPA (Solanum tuberosum) var. 'ÚNICA'
}

\author{
Patricia Martínez, Fiorela Peña, Yelena Gómez, Guino Vargas, Carmen Velezmoro*
}

\begin{abstract}
RESUMEN
La acetilación es una modificación química de la estructura del almidón que consiste en reemplazar un determinado número de grupos hidroxilos por grupos acetilos empleando anhídrido acético. Este proceso depende de ciertos factores, tales como la fuente de almidón, la concentración del anhídrido acético, el tiempo de reacción y el pH. El objetivo de este estudio fue caracterizar el almidón nativo (obtenido de la papa var. 'Única') y los almidones acetilados, mediante espectroscopía FT-IR, y evaluar sus propiedades fisicoquímicas (contenido de amilosa, color) y propiedades funcionales (poder de hinchamiento, solubilidad, capacidad de retención de agua, propiedades térmicas y propiedades de formación de pasta). Las muestras estudiadas fueron el almidón nativo (AN) y los almidones modificados por acetilación con diferentes concentraciones de anhídrido acético al $5 \%, 10 \%$ y $15 \%$ (AAc5\%, AAc10\% y AAc15\%). Los contenidos de amilosa aparente para el almidón nativo y almidón acetilado (AAc15\%) fueron 17,3 y 31,4\%, respectivamente. Los parámetros de color no cambiaron con la acetilación. La temperatura y entalpía de gelatinización, así como el perfil de formación de pasta, disminuyeron para los almidones acetilados con respecto al almidón nativo. Durante la prueba de formación de pasta, el almidón acetilado con 15\% de anhídrido acético presentó un bajo valor de la viscosidad máxima $(1599,4 \mathrm{mPa} \cdot \mathrm{s})$ con respecto al valor observado para el almidón nativo $(3065,8 \mathrm{mPa} \cdot \mathrm{s})$.
\end{abstract}

Palabras clave: almidón acetilado, amilosa, poder de hinchamiento, gelatinización, FT-IR.

Departamento de Ingeniería de Alimentos, Facultad de Industrias Alimentarias, Universidad Nacional Agraria La Molina, Av. La Molina s/n, La Molina, C.P. 12056, Lima, Perú.

*cevs@lamolina.edu.pe 


\title{
PHYSICOCHEMICAL, FUNCTIONAL AND STRUCTURAL PROPERTIES OF NATIVE AND ACETYLATED POTATO (Solanum tuberosum) STARCHES ISOLATED FROM 'ÚNICA' VARIETY
}

\begin{abstract}
Acetylation is a chemical modification of the starch structure that consists of replacing a certain number of hydroxyl groups with acetyl groups using acetic anhydride. This process depends on certain factors, such as starch source, acetic anhydride concentration, reaction time and $\mathrm{pH}$. The aim of this study was to characterize the native starch (isolated from a commercial potato 'Única' variety) and the acetylated starches by FT-IR spectroscopy, and evaluate their physicochemical (amylose content, color) and functional properties (swelling power, solubility, water retention capacity, thermal properties, and pasting profile). The evaluated samples were native starch (AN) and modified starches by acetylation with different concentrations of $5 \%, 10 \%$ and $15 \%$ acetic anhydride (AAc5\%, AAc10\% and AAc15\%). The apparent amylose contents for native starch and acetylated starch (AAc15\%) were 17,3 and $31,4 \%$, respectively. The color parameters did not change after the acetylation. Both, the temperature and the enthalpy of gelatinization as well as the pasting profile decreased for the acetylated starches with respect to the AN starch. During pasting test, acetylated starch (AAc15\%) showed a low value of the maximum viscosity $(1599,4 \mathrm{mPa} \cdot \mathrm{s}$ ) with respect to the value observed for native starch $(3065,8 \mathrm{mPa} \cdot \mathrm{s})$.
\end{abstract}

Key words: acetylated starch, amylose, swelling power, gelatinization, FT-IR.

\section{INTRODUCCIÓN}

El almidón es un polisacárido que se utiliza en la industria alimentaria como agente espesante, estabilizante, gelificante, formador de película, encapsulante e higroscópico, entre otros ${ }^{1,2}$. Sin embargo, el almidón en su forma nativa tiene poca aplicación debido a su baja solubilidad en disolventes orgánicos comunes que limitan su proceso en la industria alimentaria ${ }^{1,2,3}$. Estas deficiencias pueden ser superadas realizando una modificación estructural del almidón.

Se han utilizado diferentes métodos físicos, químicos y enzimáticos para la modificación del almidón nativo ${ }^{4}$. La modificación del almidón nativo por acetilación se logra al reemplazar los grupos $\mathrm{OH}$ por grupos acetilo $\left(\mathrm{CH}_{3} \mathrm{CO}\right)$ a lo largo de su estructura polimérica ${ }^{5,7}$. En la reacción de acetilación, la cantidad de grupos acetilo incorporados en la molécula de almidón, la velocidad y la eficiencia de la reacción, dependen de la naturaleza del reactivo, la concentración, $\mathrm{pH}$, la presencia de catalizador, el tiempo de reacción, el origen botánico del almidón y su tamaño, y las características estructurales del gránulo nativo ${ }^{4,7}$.

El almidón acetilado se clasifica según su grado de sustitución (GS) en bajo y alto. Los almidones acetilados con bajo GS $(<0,1)$ se usan comúnmente en la industria alimentaria 
pues confieren consistencia, textura y estabilidad ${ }^{5,9,10}$; mientras que los almidones con alto GS $(>1,0)$ se utilizan como sustitutos celulósicos de acetato termoplásticos ${ }^{7}$. El estudio de las propiedades de los almidones acetilados es importante para mejorar en las propiedades finales del alimento o algún proceso industrial ${ }^{1,7,8}$.

Algunas propiedades fisicoquímicas y funcionales de los almidones acetilados mostraron un aumento en la solubilidad de estos almidones, el poder de hinchamiento, la translucidez, y en la estabilidad de almacenamiento; se redujo la viscosidad, la temperatura de gelatinización, y se eliminó la retrogradación ${ }^{4,5,7}$. La retrogradación del almidón es el proceso que sigue a la gelatinización, es decir, se produce cuando la dispersión de almidón se enfría. En este proceso, las cadenas lineales de almidón interactúan entre sí, a través de los grupos hidroxilo, por medio de puentes de hidrógeno, formando un precipitado insoluble, y esta interacción genera que se retenga agua en los intersticios del almidón. Existen varios estudios referidos al efecto de la acetilación sobre las propiedades de los almidones de diferentes fuentes ${ }^{8}$. La acetilación del almidón ocasiona una ruptura de los gránulos, disminución relativa de la cristalinidad, así como también, la disminución de la temperatura y entalpía de gelatinización ${ }^{8}$. En otro estudio, la acetilación del almidón de taro mejoró el poder de hinchamiento del gránulo y redujo la solubilidad del almidón; aunque también disminuyeron la temperatura de formación de pasta, así como la temperatura y la entalpía de gelatinización ${ }^{8}$.

La variedad de papa 'Única', es una variedad comercial que ha sido mejorada en la Universidad Nacional Agraria La Molina (UNALM), y su contenido de amilosa se encuentra por debajo de lo que comúnmente se encuentra en los almidones de papas nativas. Sin embargo, para algunos usos industriales se necesitan obtener almidones que produzcan geles con baja viscosidad, lo que está relacionado con el contenido de amilosa en el almidón. Así, para evaluar la posibilidad de uso de almidones acetilados, obtenidos de esta variedad de papa; se planteó como objetivo estudiar algunas propiedades fisicoquímicas, funcionales y estructurales de los almidones acetilados.

\section{PARTE EXPERIMENTAL}

\section{Materiales y métodos}

Se emplearon los almidones: nativo (AN) y acetilados con anhídrido acético (AAc5\%, AAc $10 \%$, AAc15\%), el almidón AN fue extraído de una variedad comercial papa (Solanum tuberosum) var. 'Única', adquirida en la UNALM (Latitud: 124'35.72"S, Longitud: 76 56'38.9"W) (distrito La Molina, provincia Lima, región Lima, Perú) (Vargas et al..$^{9}$ ).

\section{Reactivos}

Las sustancias hidróxido de sodio ( $\mathrm{NaOH}$, CAS 1310-73-2), yoduro de potasio (KI, CAS 7681-11-0, pureza 99,5\%) y yodo resublimado ( $\mathrm{I}_{2}$, CAS 7553-56-2, pureza 99,8\%) fueron adquiridas de la empresa Merck ${ }^{\circledR}$ (Hohenbrunn, Alemania). Los polisacáridos amilosa de papa Tipo III (CAS 9005-82-7), amilopectina de papa (CAS 9037-22-3) y el solvente dimetilsulfóxido ( $\mathrm{C}_{2} \mathrm{H}_{6} \mathrm{OS}$, CAS 67-68-5, pureza 99,9\%) fueron adquiridos de Sigma-Aldrich 
(St. Louis, MO, USA). El agua destilada fue utilizada para la preparación de los geles de almidón y preparación de las soluciones empleadas.

\section{Equipos}

Agitador magnético (HEIDOLPH, modelo MR Hei-Tec., Alemania), agitador Vortex (VELP ${ }^{\circledR}$, modelo Wizard, Scientific, USA), balanza analítica (OHAUS ${ }^{\circledR}$, modelo Explorer, USA), baño de agua con agitación (GFL, modelo 1083, Alemania), centrífuga (HETTICH, modelo Rotofix 32, Alemania), espectrocolorímetro (KONICA MINOLTA, modelo CM-5,

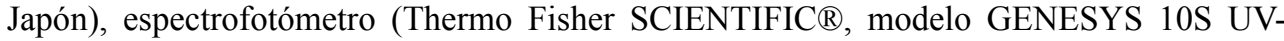
Vis, USA), espectrofotómetro infrarrojo con Transformada de Fourier equipado (FT-IR) con atenuador de reflactancia total (Thermo Scientific Inc., modelo Nicolet iS10, USA), estufa (MMM, modelo Venticell55, Alemania), multicalorímetro diferencial de barrido con celdas de acero inoxidable Hastelloy® (TA Instruments modelo Multi-Cell DSC, USA), reómetro híbrido con celda de formación de pasta de almidón (SPC) y geometría tipo rotor (TA Instruments, modelo DHR-3, USA), y refrigeradora-congeladora (GENERAL ELECTRIC®, China).

\section{Metodología}

\section{Contenido de amilosa aparente}

Se utilizó el método colorimétrico de yoduro descrito por McGrance et al. ${ }^{10}$ con algunas modificaciones, en el que se relaciona el color característico de la reacción de amilosa y amilopectina con el reactivo de yodo $\left(\mathrm{I}_{2}-\mathrm{KI}\right)$. Se pesó $20 \mathrm{mg}$ de almidón (base seca) en un tubo de ensayo de $15 \mathrm{~mL}$ y se añadió $8 \mathrm{~mL}$ de dimetilsulfóxido hasta su disolución, durante $15 \mathrm{~min}$, en un baño de agua con agitación a $85 \pm 0,2^{\circ} \mathrm{C}$. Luego, esta solución se colocó dentro de un matraz aforado y se diluyó con agua destilada hasta $50 \mathrm{~mL}$. De esta disolución, se tomó $50 \mu \mathrm{L}$ y se colocó dentro de un matraz aforado donde se adicionó $5 \mathrm{~mL}$ de solución de $\mathrm{I}_{2}(0.0025 \mathrm{M})$-KI $(0.0065 \mathrm{M})$ y se enrasó el matraz con agua destilada hasta $50 \mathrm{~mL}$ con agitación constante. Se extrae una muestra de esta solución y se lleva a un espectrofotómetro para obtener la absorbancia de la muestra a una longitud de onda de $600 \mathrm{~nm}$. Previamente, se realizaron lecturas de absorbancia de mezclas de amilosa y amilopectina puras de papa, y con un contenido de amilosa de $0,10,15,20,25,30$ y $35 \%$ en masa, con la finalidad de determinar la cantidad de amilosa aparente con ayuda de una curva patrón preparada.

\section{Evaluación del color}

Se utilizó el método descrito por Dufossé et al. ${ }^{11}$ empleando el espectrocolorímetro previamente calibrado. Se colocó el portamuestra cilíndrico $(58 \mathrm{~mm}$ de diámetro y $15 \mathrm{~mm}$ de profundidad) que contenía una cantidad de muestra de almidón sobre el puerto de luz (50 $\mathrm{mm}$ de diámetro). La información dada por los parámetros $\mathrm{L}^{*}, \mathrm{a}^{*}, \mathrm{~b}^{*}$ y $\Delta \mathrm{E}$ se expresa generalmente como el color total de las muestras en polvo, representando $\mathrm{L}^{*}$ la luminosidad, $a^{*}$ el cambio de rojo a verde y $b^{*}$ el cambio del amarillo al azul.

\section{Poder de hinchamiento, solubilidad y capacidad de absorción de agua}

Para la determinación del poder de hinchamiento $(\mathrm{PH})$, solubilidad $(\mathrm{S})$ y capacidad de absorción de agua (CAA) se siguió el método descrito por Gani et al. ${ }^{12}$, para lo cual se pesó 
0,6 g $\left(\mathrm{M}_{0}\right)$ de muestra de almidón y se mezcló con $30 \mathrm{~mL}$ de agua destilada. La suspensión se agitó y se colocó en un baño de agua durante $30 \min$ a $60,70,80$ y $90{ }^{\circ} \mathrm{C}$. Después, se enfrió la muestra a temperatura ambiente y se centrifugó a $1500 \times \mathrm{g}$ durante $30 \mathrm{~min}$. El sobrenadante se retiró cuidadosamente y el sedimento de almidón hinchado se pesó $\left(\mathrm{M}_{1}\right)$. El sobrenadante se colocó en placas Petri, y luego se llevó a la estufa a $90^{\circ} \mathrm{C}$ durante $4 \mathrm{~h}$, hasta obtener un peso constante $\left(\mathrm{M}_{2}\right)$. Los valores del poder de hinchamiento, solubilidad y la capacidad de absorción de agua se calcularon a partir de las siguientes ecuaciones:

$$
\begin{gathered}
P H=\frac{M_{1} \text { gel }(g)}{\left(M_{0} \text { almidón }-M_{2} \text { sobrenadante seco }\right)(g)} \\
S(\%)=\frac{M_{2} \text { sobrenadante } \operatorname{seco}(g)}{M_{0} \text { almidón }(g)} \times 100 \\
C A A=\frac{M_{1} \text { gel }(g)}{M_{0} \text { almidón }(g)}
\end{gathered}
$$

\section{Propiedades térmicas}

Las propiedades térmicas de gelatinización del almidón se determinaron mediante el uso del calorímetro diferencial de barrido según la metodología descrita por Lin et al. ${ }^{13}$. Se pesó aproximadamente $20 \mathrm{mg}$ de almidón en cada celda y se añadió agua ultra pura con una micropipeta (v/v, almidón:agua $=1: 3$ ), luego se sellaron herméticamente las celdas y se equilibraron a temperatura ambiente durante $1 \mathrm{~h}$ para permitir la hidratación completa del almidón. Las celdas con las muestras se calentaron desde 25 hasta $115^{\circ} \mathrm{C}$ con una velocidad de calentamiento de $2^{\circ} \mathrm{C} / \mathrm{min}$, y usando una celda vacía como referencia. Los datos obtenidos fueron: la temperatura inicial (To), temperatura de pico (Tp), temperatura de conclusión (Tc) y la entalpía de gelatinización $(\Delta \mathrm{H})$.

\section{Propiedades de formación de pasta}

El perfil de formación de pasta fue determinado según el método de la AACC14 con algunas modificaciones. La suspensión preparada de almidón al $8 \%(\mathrm{p} / \mathrm{p}$, base seca) se mantuvo por 2 min a $40^{\circ} \mathrm{C}$, luego, esta suspensión fue calentada hasta $95^{\circ} \mathrm{C}$ a $6^{\circ} \mathrm{C} / \mathrm{min}$, durante $8 \mathrm{~min}$. Finalmente, la pasta fue enfriada hasta $50^{\circ} \mathrm{C} \mathrm{a} 6^{\circ} \mathrm{C} / \mathrm{min}$, manteniéndose a $50^{\circ} \mathrm{C}$ durante $2 \mathrm{~min}$. La velocidad de agitación fue constante e igual $160 \mathrm{rpm}$.

\section{Espectroscopía Infrarrojo con Transformada de Fourier (FT-IR) con Atenuador de Reflactancia (ATR)}

Los espectros registrados de cada almidón fueron analizados usando la metodología informada por Hoyos-Leyva et al.15 dentro del rango espectral de número de onda desde 400 hasta $4000 \mathrm{~cm}-1$ con una resolución de $4 \mathrm{~cm}-1$ y una velocidad de 200 escaneos por muestra. Previo al análisis, las muestras fueron acondicionadas a $23 \pm 2{ }^{\circ} \mathrm{C}$ y $0 \%$ humedad relativa por 10 días en un desecador. Los espectros fueron obtenidos empleando el software OMNIC v.9 para la creación de los diagramas espectrales. 


\section{Análisis estadístico}

Todos los ensayos fueron realizados por triplicado, con preparación individual como ha sido descrito en cada método. Los datos obtenidos se expresaron como media \pm desviación estándar. El análisis de varianza se utilizó para calcular las diferencias significativas y se usó la prueba de discriminación de diferencias múltiples de Fisher, usando $p<0,05$ como nivel de significancia. El paquete estadístico Statgraphics CENTURION XV (Statgraphics Corp., Rockville, MD) fue utilizado para analizar los datos.

\section{RESULTADOS Y DISCUSIÓN}

\section{Contenido de amilosa aparente}

El contenido de amilosa aparente para los almidones nativo y acetilados presentaron diferencias significativas $(\mathrm{p}<0,05)$ (tabla 1). El almidón nativo de papa 'Única' presentó un contenido de amilosa de 17,3\%. Este valor obtenido fue menor a los valores registrados en almidones de papas nativas de Cusco $(24,34-29,11 \%)$, informados por Martínez et al. ${ }^{16}$, $\mathrm{y}$, además, el porcentaje de amilosa que evidenció el almidón de papa variedad 'Única' se encuentra dentro del rango de los valores obtenidos en almidones de diferentes cultivares de papa de India $(15,0-23,1 \%)$ informado por Singh et al. ${ }^{1}$.

Tabla 1. Contenido de amilosa aparente (\%) en los almidones de papa nativa y acetilados.

\begin{tabular}{|c|c|c|c|}
\hline Almidón & $\begin{array}{c}\text { Grupos acetilo } \\
(\%)(*)\end{array}$ & $\begin{array}{c}\text { Grado de } \\
\text { sustitución }(*)\end{array}$ & $\begin{array}{c}\text { Amilosa } \\
\text { aparente (\%) }\end{array}$ \\
\hline $\mathrm{AN}$ & -- & -- & $17,3 \pm 0,17^{\mathrm{a}}$ \\
\hline AAc $5 \%$ & $0,44 \pm 0,02^{\mathrm{a}}$ & $0,02 \pm 0,00^{\mathrm{a}}$ & $24,4 \pm 0,40^{\mathrm{b}}$ \\
\hline AAc $10 \%$ & $0,87 \pm 0,02^{\mathrm{b}}$ & $0,03 \pm 0,00^{\mathrm{b}}$ & $29,7 \pm 0,15^{\mathrm{c}}$ \\
\hline AAc $15 \%$ & $1,26 \pm 0,01^{\mathrm{c}}$ & $0,05 \pm 0,00^{\mathrm{c}}$ & $31,4 \pm 0,26^{\mathrm{d}}$ \\
\hline
\end{tabular}

La tabla 1 muestra que el contenido de amilosa aparente en los almidones AAc5\%, AAc10\%, y AAc15\% aumentó según el número de los grupos acetilo formados (el símbolo \% representa el porcentaje en peso de grupos acetilo en el almidón en base seca) y según el grado de sustitución (número promedio de sitios sustituidos por unidad de glucosa) en el almidón modificado. Este resultado fue similar a los informados por Mbougueng et al. ${ }^{18}$, con respecto a los almidones acetilados de papa Sipiera que registraron mayores contenidos de amilosa aparente $(39,16-41,11 \%)$, y por Singh et al. ${ }^{17}$ con respecto al almidón acetilado de papa comercial con menores contenidos de amilosa aparente (19,5-22,9\%). Betancur et al. ${ }^{4}$ sostienen que este incremento en el contenido de amilosa aparente podría deberse a que los grupos acetilo formados en las cadenas de almidón interfieren con la reagrupación de las fracciones amilosa y amilopectina durante el enfriamiento de las moléculas de almidón que han sido sometidas a un proceso de gelatinización. Tal es así que, la creación de más segmentos lineales (amilosa) facilita la absorción de una mayor cantidad de yodo, que se refleja en el contenido de amilosa aparente obtenida, que depende del número de grupos acetilo formados. 


\section{Determinación de color}

Los parámetros de color $\mathrm{L}^{*}, \mathrm{a}^{*}, \mathrm{y} \mathrm{b}^{*}$ de los almidones nativo y acetilados se presentan en la tabla 2. El color blanco es una propiedad física primordial de los almidones y en muchas aplicaciones industriales esta característica es muy significativa, como parámetro de calidad; en general, se busca que los almidones puedan ser utilizados en las formulaciones de productos sin impartir un color adverso (Joaqui y Villada ${ }^{19}$ ).

Tabla 2. Efecto de la acetilación sobre los parámetros de color en los almidones nativo y acetilados.

\begin{tabular}{|c|c|c|c|}
\hline Almidón & $\begin{array}{c}\text { Grupos acetilo } \\
(\%)(*)\end{array}$ & $\begin{array}{c}\text { Grado de } \\
\text { sustitución (*) }\end{array}$ & $\begin{array}{c}\text { Amilosa } \\
\text { aparente }(\%)\end{array}$ \\
\hline $\mathrm{AN}$ & - & -- & $17,3 \pm 0,17^{\mathrm{a}}$ \\
\hline AAc $5 \%$ & $0,44 \pm 0,02^{\mathrm{a}}$ & $0,02 \pm 0,00^{\mathrm{a}}$ & $24,4 \pm 0,40^{\mathrm{b}}$ \\
\hline AAc $10 \%$ & $0,87 \pm 0,02^{b}$ & $0,03 \pm 0,00^{\mathrm{b}}$ & $29,7 \pm 0,15^{\mathrm{c}}$ \\
\hline AAc $15 \%$ & $1,26 \pm 0,01^{\mathrm{c}}$ & $0,05 \pm 0,00^{\mathrm{c}}$ & $31,4 \pm 0,26^{\mathrm{d}}$ \\
\hline
\end{tabular}

Los resultados de la evaluación del color en los almidones de papa nativo y acetilados indican que existen diferencias significativas $(\mathrm{p}<0,05)$. El valor de $\mathrm{L}^{*}$ para el almidón de papa nativo fue de 95,35. Este valor fue menor con respecto a los valores $\mathrm{L}^{*}$ encontrados en este trabajo para los almidones AAc5\%, AAc10\% y AAc15\% (96,79-96,83), ligeramente mayor al informado por Das et al. ${ }^{20}(93,89)$ para almidón nativo de camote de India y se encuentra dentro del rango de los valores $L^{*}(94,28-96,67)$ informados por Das et al. ${ }^{20}$ para almidones de camote acetilados con vinil acetato.

El valor de $a^{*}$ para el almidón nativo de papa fue igual a -0,12 mientras que los valores de $\mathrm{a}^{*}$ para almidones acetilados (AAc5\%, AAc10\% y AAc15\%), estuvieron en el rango de $-0,16$ y $-0,23$ con diferencias significativas $(\mathrm{p}<0,05)$. Sin embargo, Das et al. ${ }^{20}$ informaron valores positivos de $\mathrm{a}^{*}(0,17-0,22)$ encontrados para almidones de camote acetilados con vinil acetato. Los valores negativos de a* ubican a estos almidones en la fracción verde, y por estar cercano al valor cero, tienen más tendencia a un color neutro (Joaqui y Villada ${ }^{19}$ ). Por otro lado, los valores de $b^{*}$ oscilaron entre 1,62 y 2,90, para los almidones acetilados (AAc5\%, AAc10\% y AAc15\%) y el almidón nativo presentó un valor de b* igual a 3,45; estos valores también presentaron diferencias significativas $(\mathrm{p}<0,05)$. Das et al.$^{20}$ informaron mayores valores de $b^{*}(3,06-3,27)$ para almidones acetilados y un valor de $b^{*}$ igual a 3,32 para el almidón nativo. Los valores positivos de $b^{*}$ indican una ligera tendencia al color amarillo.

\section{Poder de hinchamiento, solubilidad y capacidad de absorción de agua}

El aumento de los valores del poder de hinchamiento (PH) (tabla 3) de los almidones acetilados, observado a diferentes temperaturas, puede atribuirse a la presencia de los 
grupos acetilo formados como resultado de la modificación química estructural realizada. Esta modificación altera la estructura del almidón, ocasionando la ruptura de los enlaces de hidrógeno y permitiendo mayor ingreso del agua en el almidón, lo que finalmente resulta en un mayor PH del almidón acetilado (figura 1) (Betancur et al. ${ }^{4}$, Ali \& Hasnain ${ }^{21}$ ).

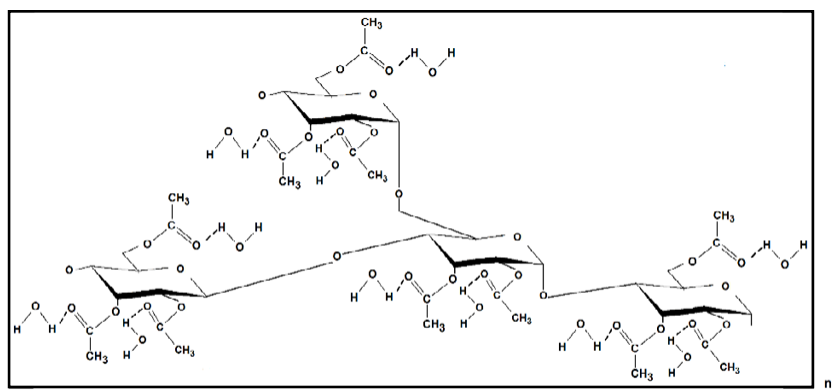

Figura 1. Fórmula estructural del almidón acetilado y sus probables enlaces de hidrógeno.

Para el almidón nativo, el valor de $\mathrm{PH}$ a $90^{\circ} \mathrm{C}(15,86 \mathrm{~g} / \mathrm{g})$ fue menor con respecto a los valores de $\mathrm{PH}$ evaluados en almidones de papas nativas de Cusco $(23,9-28,5 \mathrm{~g} / \mathrm{g})^{16}$, en almidones de papas de la India $(58,12-70,15 \mathrm{~g} / \mathrm{g})^{1}$ y almidón de papa de la India $(20,3 \mathrm{~g} / \mathrm{g})^{17}$. Con respecto a los almidones acetilados, Singh et al. ${ }^{1}$ informaron acerca de valores muy altos de PH encontrados $(64,15-70,58 \mathrm{~g} / \mathrm{g})$ mientras que Singh et al. ${ }^{17}$ informaron acerca de valores de PH (22,8-34,4 g/g) cercanos a los encontrados en este estudio (18,36-21,62 g/g).

Tabla 3. Poder de hinchamiento ( $\mathrm{PH})$, solubilidad (S) y capacidad de absorción de agua (CAA) en los almidones nativo y acetilados.

\begin{tabular}{|c|c|c|c|c|}
\hline Temperatura $\left({ }^{\circ} \mathrm{C}\right)$ & Almidón (GS)* & PH (g/g) & S (\%) & $\mathbf{C A A}(\mathrm{g} / \mathrm{g})$ \\
\hline \multirow{4}{*}{60} & $\mathrm{AN}(0)$ & $4,78 \pm 0,06^{\mathrm{a}}$ & $1,52 \pm 0,28^{\mathrm{a}}$ & $4,70 \pm 0,05^{\mathrm{a}}$ \\
\hline & AAc $5 \%(0,02)$ & $9,54 \pm 0,10^{\mathrm{b}}$ & $3,29 \pm 0,62^{\mathrm{b}}$ & $9,23 \pm 0,04^{\mathrm{b}}$ \\
\hline & AAc $10 \%(0,03)$ & $11,13 \pm 0,28^{\mathrm{c}}$ & $4,08 \pm 1,03^{\mathrm{b}}$ & $10,68 \pm 0,38^{\mathrm{c}}$ \\
\hline & AAc $15 \%(0,05)$ & $12,56 \pm 0,02^{\mathrm{d}}$ & $4,80 \pm 0,05^{\mathrm{b}}$ & $11,96 \pm 0,01^{\mathrm{d}}$ \\
\hline \multirow{4}{*}{70} & AN (0) & $13,32 \pm 1,12^{\mathrm{a}}$ & $4,73 \pm 0,06^{\mathrm{a}}$ & $12,69 \pm 1,05^{\mathrm{a}}$ \\
\hline & AAc $5 \%(0,02)$ & $13,67 \pm 0,03^{\mathrm{a}}$ & $6,14 \pm 0,13^{b}$ & $12,83 \pm 0,04^{\mathrm{a}}$ \\
\hline & AAc $10 \%(0,03)$ & $14,00 \pm 0,14^{\mathrm{ab}}$ & $8,14 \pm 0,61^{\mathrm{c}}$ & $12,86 \pm 0,04^{\mathrm{a}}$ \\
\hline & AAc15\% $(0,05)$ & $15,30 \pm 0,24^{\mathrm{b}}$ & $9,67 \pm 0,11^{\mathrm{d}}$ & $13,82 \pm 0,24^{\mathrm{a}}$ \\
\hline \multirow{4}{*}{80} & AN (0) & $15,22 \pm 0,09^{\mathrm{a}}$ & $6,81 \pm 0,02^{\mathrm{a}}$ & $14,18 \pm 0,08^{\mathrm{a}}$ \\
\hline & AAc $5 \%(0,02)$ & $15,57 \pm 0,38^{\mathrm{a}}$ & $7,41 \pm 0,44^{\mathrm{a}}$ & $14,42 \pm 0,28^{\mathrm{a}}$ \\
\hline & AAc $10 \%(0,03)$ & $16,50 \pm 0,71^{\mathrm{ab}}$ & $10,17 \pm 0,52^{\mathrm{b}}$ & $14,82 \pm 0,72^{\mathrm{a}}$ \\
\hline & AAc15\% $(0,05)$ & $17,17 \pm 0,45^{\mathrm{b}}$ & $10,29 \pm 0,20^{\mathrm{b}}$ & $15,41 \pm 0,44^{\mathrm{a}}$ \\
\hline \multirow{4}{*}{90} & $\mathrm{AN}(0)$ & $15,86 \pm 0,20^{\mathrm{a}}$ & $8,94 \pm 0,20^{\mathrm{a}}$ & $14,44 \pm 0,15^{\mathrm{a}}$ \\
\hline & AAc $5 \%(0,02)$ & $18,36 \pm 0,04^{\mathrm{b}}$ & $11,56 \pm 0,93^{\mathrm{b}}$ & $16,24 \pm 0,14^{\mathrm{b}}$ \\
\hline & AAc10\% $(0,03)$ & $20,81 \pm 0,86^{\mathrm{c}}$ & $13,61 \pm 0,60^{\mathrm{c}}$ & $17,98 \pm 0,62^{\mathrm{c}}$ \\
\hline & AAc $15 \%(0,05)$ & $21,62 \pm 0,57^{\mathrm{c}}$ & $14,07 \pm 0,43^{\mathrm{c}}$ & $18,58 \pm 0,59^{\mathrm{c}}$ \\
\hline
\end{tabular}


Los resultados de la solubilidad (S) del almidón nativo y de los almidones acetilados presentaron diferencias significativas $(p<0,05)$ (tabla 3 ). A medida que se incrementa el grado de acetilación aumenta la solubilidad del almidón en el agua, debido a una mayor captación de moléculas de agua y a la ruptura de los enlaces de hidrógeno que produce una mayor movilidad de las cadenas poliméricas (amilosa y amilopectina) y la lixiviación de la amilosa (Ali \& Hasnain ${ }^{21}$ ). Estos valores tendrían una correlación significativa con el contenido de los grupos acetilos formados en almidones de papa acetilados (AAc5\%, AAc10\%, AAc15\%). Para el almidón nativo, el valor de $\mathrm{S}$ a $90^{\circ} \mathrm{C}(8,94 \%)$ fue más bajo con respecto a los valores de S informados por Singh et al. ${ }^{1}$ (10,2-19,2 \%) para almidones de papas de la India y por Martínez et al. ${ }^{16}(16,4-20,8 \%)$ para almidones de papas nativas de Cusco. Sin embargo, el valor de valor de $\mathrm{S}$ encontrado para el almidón nativo fue mayor al valor de $\mathrm{S}(5 \%)$ encontrado para el almidón de papa de la India informado por Singh et al. ${ }^{17}$.

La capacidad de absorción de agua (CAA) del almidón nativo y de los almidones acetilados presentaron diferencias significativas $(p<0,05)$ a 60 y $90^{\circ} \mathrm{C}$ (tabla 3$)$. En este estudio, los valores de CAA para los almidones acetilados son mayores que para el almidón nativo, y aumentaron con el mayor grado de sustitución. Esto demuestra que la tendencia hidrofílica del almidón aumentó con la acetilación, pues se asegura una alta retención de agua que ingresa al gránulo, aumenta el poder de hinchamiento y favorece la gelatinización (Betancur et al. ${ }^{4}$, Mbougueng et al. $\left.{ }^{18}\right)$. El valor de CAA a $90^{\circ} \mathrm{C}(14,44 \mathrm{~g} / \mathrm{g})$ obtenido en el almidón nativo, fue menor con respecto a los valores valor de CAA encontrados en almidones de papas nativas de Cusco (18,9-22,7 g/g), informados por Martínez et al. ${ }^{16}$, y en almidón nativo de papa de Irlanda (42 $\mathrm{g} / \mathrm{g}$ ), informados por Mbougueng et al. ${ }^{18}$. Los valores de CAA encontrados para los almidones acetilados a $90^{\circ} \mathrm{C}(16,24-18,58 \mathrm{~g} / \mathrm{g})$ son menores a los valores de CAA encontrados para almidón acetilado de papa 2425 de Irlanda $(40 \mathrm{~g} / \mathrm{g}$ ) informados por Mbougueng et al. ${ }^{18}$.

\section{Propiedades térmicas de los geles de almidón}

Las propiedades térmicas de los geles de almidones nativo y acetilados presentaron diferencias significativas $(p<0,05)$ (tabla 4). Las temperaturas $\left(\mathrm{T}_{\mathrm{o}}, \mathrm{T}_{\mathrm{p}} \mathrm{y} \mathrm{T}_{\mathrm{c}}\right)$ y la entalpía de gelatinización $\left(\Delta \mathrm{H}_{\mathrm{g}}\right)$ disminuyeron a medida que aumentó el número de grupos acetilo formados, de tal forma que el almidón nativo presentó temperaturas de gelatinización y entalpía más altas que las de los almidones acetilados; lo que coincide con la modificación por acetilación de almidones nativos de diversas fuentes botánicas (Sánchez-Rivera et al. ${ }^{6}$, Singh et al. ${ }^{17}$; Wang y Wang $^{5}$ ). 
Propiedades fisicoquímicas, funcionales y estructurales de almidones nativos y acetilados obtenidos a partir.. 347

Tabla 4. Temperaturas y entalpía de gelatinización de los almidones nativo y acetilados.

\begin{tabular}{lcccc}
\hline Almidón & $\mathbf{T}_{\mathbf{o}}\left({ }^{\circ} \mathbf{C}\right)$ & $\mathbf{T}_{\mathbf{p}}\left({ }^{\circ} \mathbf{C}\right)$ & $\mathbf{T}_{\mathbf{c}}\left({ }^{\circ} \mathbf{C}\right)$ & $\Delta \mathbf{H}_{\mathbf{g}}(\mathbf{J} / \mathbf{g})^{*}$ \\
\hline AN & $62,28 \pm 0,26^{\mathrm{c}}$ & $65,79 \pm 0,25^{\mathrm{c}}$ & $72,44 \pm 0,26^{\mathrm{d}}$ & $15,16 \pm 0,18^{\mathrm{b}}$ \\
AAc5\% & $59,79 \pm 0,12^{\mathrm{c}}$ & $64,49 \pm 0,16^{\mathrm{b}}$ & $70,31 \pm 0,15^{\mathrm{c}}$ & $13,55 \pm 0,93^{\mathrm{a}}$ \\
AAc10\% & $59,73 \pm 0,04^{\mathrm{b}}$ & $64,30 \pm 0,11^{\mathrm{b}}$ & $69,86 \pm 0,01^{\mathrm{b}}$ & $13.51 \pm 0,04^{\mathrm{a}}$ \\
AAc15\% & $58,28 \pm 0,28^{\mathrm{a}}$ & $62,47 \pm 0,17^{\mathrm{a}}$ & $69,28 \pm 0,15^{\mathrm{a}}$ & $13,35 \pm 0,28^{\mathrm{a}}$ \\
\hline *en base seca \\
$\begin{array}{l}\text { Valores promedio } \pm \text { desviación estándar; valores en la misma columna con diferentes letras son } \\
\text { significativamente diferentes }(p<0,05) .\end{array}$
\end{tabular}

Tal como se observa en la tabla 4, la temperatura de gelatinización fue menor en el almidón AAc $15 \%$ con respecto a los otros almidones acetilados (AAc5\% y AAc10\%). Estos resultados indican una desorganización de la doble hélice de la amilopectina (Núñez-Santiago et al. ${ }^{3}$ ) y una menor estabilidad del almidón después de la acetilación (Ali \& Hasnain ${ }^{21}$ ). Esta menor estabilidad de los almidones acetilados se atribuye a la desorganización del ensamblaje interno del almidón debido a la pérdida de las fuerzas de atracción intramolecular e intermolecular, lo que resulta en una pérdida eventual de la región cristalina que conduce a una hidratación y gelatinización más fácil de los gránulos de almidón químicamente modificados, y por consiguiente una disminución de la temperatura de gelatinización (Ali \& Hasnain ${ }^{21}$, Núñez-Santiago et al. ${ }^{3}$, Singh et al. ${ }^{17}$, Wang y Wang ${ }^{5}$ ). El almidón acetilado AAc15\% (GS $0,05)$, presentó la entalpía de gelatinización más baja comparada con los demás almidones AAc5\% y AAc10\%. Este resultado puede atribuirse a la presencia de los grupos acetilos y al incremento en el número de enlaces de hidrógeno en las moléculas de almidón, lo que favoreció la gelatinización a baja temperatura (Singh et al. ${ }^{17}$ ).

Mbougueng et al. ${ }^{18}$ afirmaron que la disminución de la temperatura de gelatinización del almidón acetilado con respecto al almidón nativo es una de las numerosas ventajas conseguidas con la modificación del almidón por acetilación, lo que permite emplear a estos derivados en procesos cuando un agente espesante debe gelificarse a temperaturas más bajas o simplemente reducir los costos energéticos durante la fabricación de los productos donde se utilizan estos derivados. Los resultados obtenidos son similares a los informados por Singh et al. ${ }^{1}$ para almidones de cuatro variedades de papa de India, por Simsek et al. ${ }^{8}$ para almidones de dos variedades de frijol de México, y por Singh et al. ${ }^{17}$ para almidón de papa de India empleando diferentes concentraciones de anhídrido acético.

\section{Propiedades de formación de pasta}

Cuando la suspensión de almidón se calienta, la viscosidad aumenta gradualmente hasta alcanzar un valor máximo, denominada viscosidad máxima, que representa el período desde el hinchamiento de los gránulos hasta la ruptura de la red de almidón (Núñez-Santiago et al. ${ }^{3}$, Sánchez-Rivera et al. ${ }^{6}$, Bello-Pérez et al. ${ }^{7}$ ). En la figura 2 se observa el perfil de formación de pasta de los almidones estudiados, donde la curva continua observada en la parte superior, indica el cambio de temperatura durante las etapas de calentamiento, mantenimiento y 
enfriamiento en la formación de la pasta. Cuando el almidón se calienta en exceso de agua, sus gránulos se hinchan y al mismo tiempo, parte de sus componentes (amilosa y amilopectina) se solubilizan, dando lugar a una suspensión de partículas hinchadas y dispersas en una fase continua. A la concentración ensayada ( $8 \%, \mathrm{p} / \mathrm{p}$, base seca), el valor máximo de viscosidad del almidón $\mathrm{AN}$ fue $3065,8 \mathrm{mPa} \cdot \mathrm{s}$ a una temperatura de $90^{\circ} \mathrm{C}$; comportamiento similar presentaron los almidones AAc5\%, AAc10\%, y AAc15\%, con menores valores de viscosidad máxima $(2066,7 ; 2006,6$ y 1599,4 $\mathrm{mPa} \cdot \mathrm{s}$, respectivamente). Se pudo observar que a una acetilación del $15 \%$, la máxima viscosidad de formación de pasta se redujo a la mitad con respecto a la viscosidad del almidón nativo.

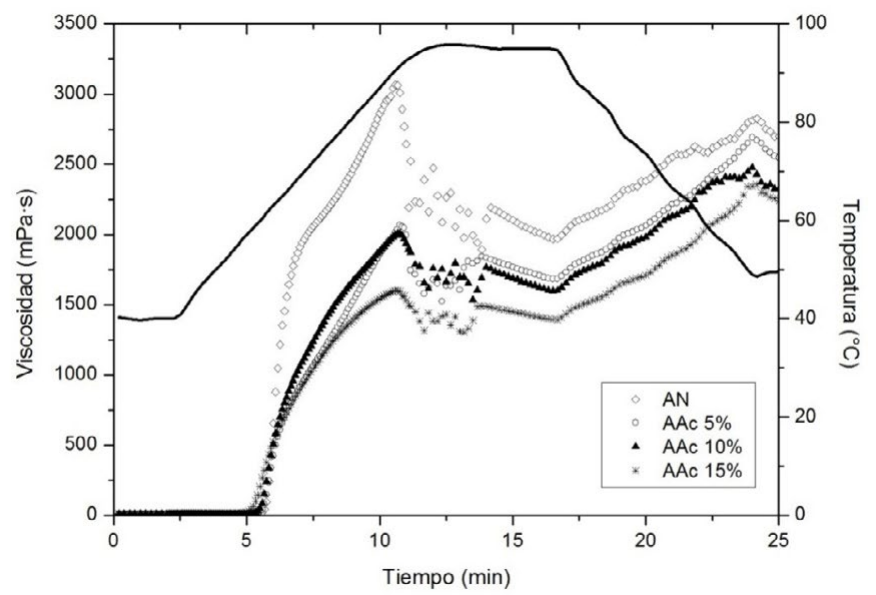

Figura 2. Perfil de formación de pasta de los almidones nativo y acetilados.

\section{Espectroscopía Infrarrojo por Transformada de Fourier (FT-IR) con atenuador de reflactancia (ATR)}

La espectroscopía FT-IR se utiliza para evaluar la organización de la región externa de los gránulos de almidón; el modo ATR se reconoce como un método de análisis de superficie, pues el haz infrarrojo penetra aproximadamente $2,0 \mathrm{~mm}$ en la superficie del gránulo de almidón (Hoyos-Leyva et al. ${ }^{15}$ ). La presencia de los grupos acetilo formados en los almidones modificados fue evidenciado mediante esta técnica espectroscópica. Los espectros de FTIR de los almidones nativo y acetilados (figura 4) muestran bandas anchas de absorción en el rango de $3000-3700 \mathrm{~cm}^{-1}$ y $1641,57-1644,02 \mathrm{~cm}^{-1}$ correspondientes a las vibraciones de estiramiento y de flexión, respectivamente, de los grupos hidroxilos $(\mathrm{OH})$ presentes en las unidades de glucosa que conforman las cadenas de amilosa y amilopectina. Estas bandas de absorción son similares a las bandas IR informadas por Hoyos-Leyva et al. ${ }^{15}$, Colussi et al. ${ }^{23} \mathrm{y}$ Shah et al. ${ }^{22}$. Además, en todos los espectros FT-IR se observa una señal cercana a $2928 \mathrm{~cm}^{-1}$ que corresponde a la frecuencia de estiramiento asimétrico del grupo metileno $(-\mathrm{CH} 2)$ (Shah et al. $^{22}$ ) y una banda ancha en el rango de $993,33-994,60 \mathrm{~cm}^{-1}$ asignada a las frecuencias de estiramientos de los enlaces $\mathrm{C}-\mathrm{OH}\left(\mathrm{Chi}\right.$ et $\left.a l .^{2}\right)$. 


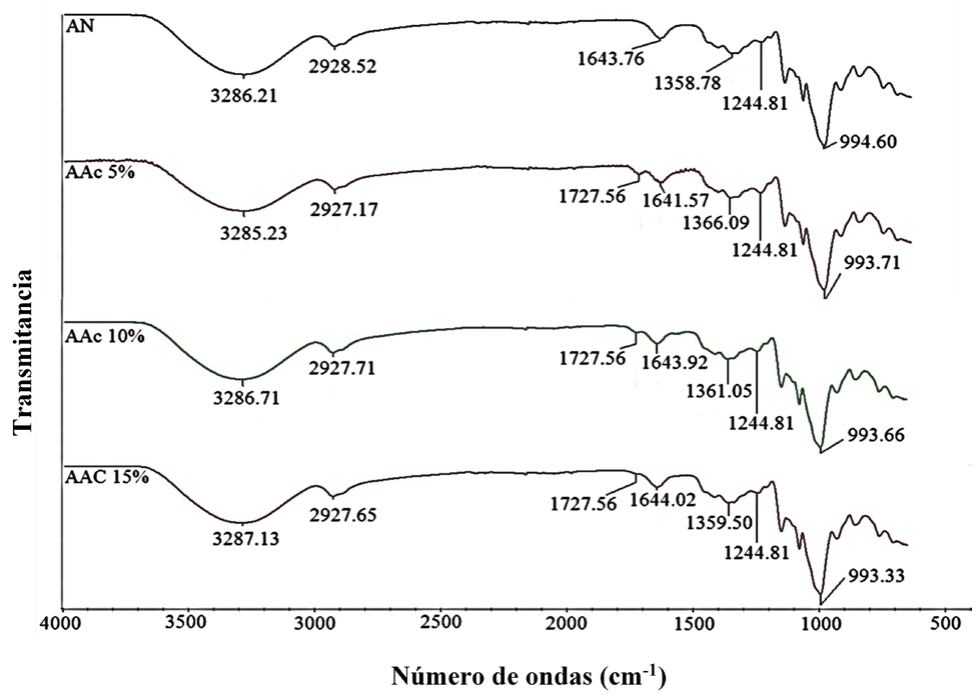

Figura 3. Espectros FT-IR para los almidones nativo y acetilados.

Los espectros FT-IR para los almidones AAc5\%, AAc10\%, y AAc15\%, mostraron una nueva señal a $1727,56 \mathrm{~cm}^{-1}$ correspondiente a la vibración del grupo carboxilato (-COO-). Estas evidencias encontradas fueron similares a los resultados informados por Shah et al. ${ }^{22}$ y Colussi et al. ${ }^{23}$. La aparición de bandas de absorción en el rango de $1358-1366 \mathrm{~cm}^{-1} \mathrm{y}$ a $1244,81 \mathrm{~cm}^{-1}$, fueron asignadas a la vibración simétrica de los grupos $\mathrm{CH}_{3}$ y las vibraciones de estiramiento del enlace $\mathrm{C}-\mathrm{O}$, respectivamente; valores similares fueron informados por Shah et al. ${ }^{22} \mathrm{y}$ Colussi et al..$^{23}$. Además, Chi et al. ${ }^{2}$ y Mbougueng et al. ${ }^{18}$ informaron que estos resultados confirmarían la presencia de grupos acetilos en los almidones modificados.

\section{CONCLUSIONES}

Mediante la espectroscopía FT-IR, se confirmó la presencia de los grupos acetilos en la estructura de los almidones modificados. Los almidones de papa acetilados mostraron un aumento en el contenido aparente de amilosa, con respecto al almidón nativo. La acetilación produjo una disminución en la viscosidad máxima durante la formación de pasta y este efecto fue mayor en los almidones acetilados con mayor porcentaje de grupos acetilos. Los almidones acetilados presentaron menores temperaturas y entalpías de gelatinización con respecto al almidón nativo; mientras que el poder de hinchamiento, solubilidad y capacidad de absorción de agua, aumentaron según el grado de sustitución de los grupos hidroxilos. Futuras investigaciones sobre la aplicación de los almidones modificados en alimentos se deben desarrollar dependiendo de la calidad de producto que se requiera comercializar. 


\section{AGRADECIMIENTOS}

Los autores agradecen a INNÓVATE PERÚ por el apoyo financiero en los Convenios $\mathrm{N}^{\circ}$ 121-FINCYT-ECL-2014 y N 139-PNICP-PIAP-2015. De igual manera, los autores agradecen a la Mg.Sc. Lena Téllez Monzón del Centro de Investigación en Química, Toxicología y Biotecnología Ambiental (CIQTOBIA) de la Facultad de Ciencias de la Universidad Nacional Agraria La Molina, por su colaboración en el registro de los espectros FT-IR empleando el Espectrofotómetro Infrarrojo con Transformada de Fourier con Atenuador de Reflactancia.

\section{REFERENCIAS BIBLIOGRÁFICAS}

1. Singh J, Kaur L, Singh N. Effect of Acetylation on Some Properties of Corn and Potato Starches. Starch/Stärke. 2004; 56: 586-601.

2. Chi H, Xu K, Wu X, Chen Q, Xue D, Song C, Zhang W, Wang P. Effect of acetylation on the properties of corn starch. Food Chem. 2008; 106: 923-92.

3. Núñez-Santiago C, García-Suarez FJ, Román-Gutiérrez AD, Bello-Pérez LA. Effect of reagent type on the acetylation of barley and maize starches. Starch/Stärke. 2010; 62(9): 489-497.

4. Betancur AD, Chel GL, Cañizares HE. Acetylation and characterization of Canavalia ensiformis starch. J Agric Food Chem. 1997; 45: 378-382.

5. Wang Y, Wang L. Characterization of acetylated waxy maize starches prepared under catalysis by different alkali and alkaline-earth hydroxides. StarchStärke. 2002; 54: 25 30 .

6. Sánchez-Rivera MM, Flores-Ramírez I, Zamudio-Flores PB, González-Soto RA. Acetylation of banana (Musa paradisiaca L.) and maize (Zea mays L.) starches using a microwave heating procedure and iodine as catalyst: Partial characterization. Starch/ Stärke. 2010; 62:155-164.

7. Bello-Pérez L, Agama-Acevedo E, Zamudio-Flores P, Mendez-Montealvo G, Rodriguez-Ambriz S. Effect of low and high acetylation degree in the morphological, physicochemical and structural characteristics of barley starch. LWT Food Sci Technol. 2010; 43(9): 1434-1440.

8. Simsek S, Ovando-Martínez M, Whitney K. Bello-Pérez L. Effect of acetylation, oxidation and annealing on physicochemical properties of bean starch. Food Chem. 2012; 134(4): 1796-1803.

9. Vargas G, Martínez P, Velezmoro C. Propiedades funcionales de almidón de papa (Solanum tuberosum) y su modificación química por acetilación. Sci agropecu. 2016; 7(3): 223-230.

10. McGrance S, Cornell H, Rix C. A simple and rapid colorimetric method for the determination of amylose in starch products. Starch/Stärke, 1998; 50(4): 158-163.

11. Dufossé L, Mabon P, Binet A. Assessment of the coloring strength of Brevibacterium linens strains: spectrocolorimetry versus total carotenoid extraction/quantification. J Dairy Sci. 2001; 84(2): 354-360. 
12. Gani A, Nazia S, Rather SA, Wani SM, Shah A, Bashir M, Masoodi FA, Gani A. Effect of $\gamma$-irradiation on granule structure and physicochemical properties of starch extracted from two types of potatoes grown in Jammu \& Kashmir, India. LWT Food Sci Technol. 2014; 58(1): 239-246.

13. Lin J, Kao W, Tsai Y, Chang Y. Effect of granular characteristics on pasting properties of starch blends. Carbohydr Polym. 2013; 98(2): 1553-1560.

14. AACC International. Approved Methods of Analysis, 11th Ed. Method 76-21.01. General Pasting Method for Wheat or Rye Flour or Starch Using the Rapid Visco Analyser. St. Paul, MN (U.S.A.): AACC International; Approved November 3, 1999. doi: 10.1094/ AACCIntMethod-76-21.01.

15. Hoyos-Leyva JD, Alonso-Gomez L, Rueda-Enciso J, Yee-Madeira H, Bello-Perez, LA, Alvarez-Ramirez J. Morphological, physicochemical and functional characteristics of starch from Marantha ruiziana Koern. LWT Food Sci Technol. 2017; (83): 150-156.

16. Martínez P, Málaga A, Betalleluz I, Ibarz A, Velezmoro C. Caracterización funcional de almidones nativos obtenidos de papas (Solanum phureja) nativas peruanas. Sci agropecu. 2015; 6(4): 291-301.

17. Singh N, Chawla D, Singh J. Influence of acetic anhydride on physicochemical, morphological and thermal properties of corn and potato starch. Food Chem. 2004; 86(4): 601-608.

18. Mbougueng P, Tenin D, Scher J, Tchiégang C. Influence of acetylation on physicochemical, functional and thermal properties of potato and cassava starches. J Food Eng. 2012; 108(2): 320-326.

19. Joaqui D, Villada H. Propiedades ópticas y permeabilidad de vapor de agua en películas producidas a partir de almidón. Rev Bio Agro. 2013; 11(2): 59-68.

20. Das AB, Singh G, Singh S, Riar CS. Effect of acetylation and dual modification on physico-chemical, rheological and morphological characteristics of sweet potato (Ipomoea batatas) starch. Carbohydr. Polym. 2010; 80(3): 725-732.

21. Ali TM, \& Hasnain, A. Physicochemical, morphological, thermal, pasting, and textural properties of starch acetates. Food Rev Int. 2015, 32(2): 161-180.

22. Shah A, Masoodi FA, Gani A, Ahmad B. Physicochemical, rheological and structural characterization of acetylated oat starches. LWT Food Sci Technol. 2017; 80: 19-26.

23. Colussi R, El Halal SL, Pinto VZ, Bartz J, Gutkoski LC, da Rosa E, Dias AR. Acetylation of rice starch in an aqueous medium for use in food. LWT Food Sci Technol. 2015; 62(2): 1076-1082. 